\title{
Índice de área foliar, coeficiente de extinção luminosa e acúmulo de forragem em pastagens de Cynodon spp. sob lotação contínua ${ }^{(1)}$
}

\author{
Jailson Lara Fagundes ${ }^{(2)}$, Sila Carneiro da Silva(3), Carlos Guilherme Silveira Pedreira( ${ }^{(3)}$, Roberta Aparecida Carnevalli(2), \\ Carlos Augusto Brandão de Carvalho(4), Andre Fisher Sbrissia ${ }^{(4)}$ e Luis Felipe de Moura Pinto(2)
}

\begin{abstract}
Resumo - Avaliou-se o índice de área foliar, o coeficiente de extinção luminosa e o acúmulo de matéria seca em três cultivares de Cynodon spp. (Tifton 85, Florakirk e Coastcross) submetidas a quatro intensidades de pastejo $(5,10,15$ e $20 \mathrm{~cm})$ por ovinos em regime de lotação contínua. O delineamento experimental utilizado foi o de blocos completos casualizados, com quatro repetições, em parcelas subdivididas, tendo na parcela as cultivares, e na subparcela, as intensidades de pastejo. Os maiores valores de índice de área foliar foram encontrados na cultivar Tifton 85. A extinção de luz dentro do dossel do pasto variou com a cultivar, mês do ano e intensidade de pastejo. Os valores mais elevados de coeficiente de extinção de luz $(\mathrm{k})$ foram observados nos pastos mantidos mais altos, dentro de cultivares, na Florakirk. As taxas de acúmulo de matéria seca foram semelhantes em todas as cultivares avaliadas, independentemente dos regimes de desfolha adotados.
\end{abstract}

Termos para indexação: gramíneas forrageiras, ovinos, intensidade de pastejo, manejo de pastagem.

\section{Leaf area index, light extinction coefficient and herbage accumulation in Cynodon spp. swards under continuous stocking management}

\begin{abstract}
Leaf area index, light extinction coefficient and herbage accumulation were evaluated in three Cynodon spp. cultivars (Tifton 85, Florakirk and Coastcross) submitted to four grazing intensities $(5,10,15$ and $20 \mathrm{~cm})$ imposed by sheep under a continuous stocking management. The experimental design was a complete randomized blocks with four replications, with treatments in a split plot arrangement. Grass cultivars were allocated to plots and grazing intensities to subplots. The largest values of LAI were observed for Tifton 85. Light extinction in the sward was a function of grass cultivar, time of the year and phenological state of pasture plants. Florakirk presented the highest values of light extinction coefficient $(\mathrm{k})$, but $\mathrm{k}$ was higher for lower grazing intensities. No differences were observed in herbage accumulation rates among the grass plants studied.
\end{abstract}

Index terms: feed grasses, sheep, grazing intensity, grassland management.

\section{Introdução}

Ecossistemas de pastagens são entidades bastante complexas, e possuem uma série de componentes

(1) Aceito para publicação em 22 de março de 2000.

Extraído da dissertação de mestrado, apresentada pelo primeiro autor à Escola Superior de Agricultura Luiz de Queiroz (ESALQ), Piracicaba, SP.

Parcialmente financiado pela FAPESP.

${ }^{(2)}$ ESALQ, Dep. de Produção Animal, Caixa Postal 9, CEP 13418-900 Piracicaba, SP. Bolsista da CAPES.

E-mail: racarnev@carpa.ciagri.usp.br, omazzo@bol.com.br

${ }^{(3)}$ ESALQ, Dep. de Produção Animal.

E-mail: scdsilva@carpa.ciagri.usp.br, cgspedre@carpa.ciagri.usp.br

${ }^{(4)}$ ESALQ, Dep. de Produção Animal. Bolsista da FAPESP.

E-mail: sbrissia@bol.com.br bióticos e abióticos que interagem entre si de diferentes maneiras. Assim, para a devida compreensão e entendimento das respostas de plantas forrageiras em pastejo, torna-se essencial e crítico que parâmetros relacionados com a sua biologia e ecologia sejam avaliados e considerados quando da idealização de estratégias de uso das pastagens como recurso produtivo (Silva \& Pedreira, 1997). O manejo da pastagem é, na sua essência, o compromisso entre a necessidade de manter área foliar para fotossíntese e a de colher grandes quantidades de tecidos vegetais de alta qualidade, particularmente folhas, antes que estas venham a senescer e se decompor (Parsons, 1988).

Entre os métodos de utilização das culturas forrageiras, o pastejo é o mais econômico, uma vez 
que a colheita da forragem é feita pelo próprio animal (Hodgson, 1990). Contudo, segundo Jacques (1973), práticas de manejo do pastejo devem ser determinadas em razão das características morfológicas e fisiológicas das plantas forrageiras para assegurar altas produções e persistência das pastagens, além de serem governadas por características de crescimento da planta e das condições de ambiente. Diversas são as características que estão relacionadas com o manejo de forrageiras, destacando-se área foliar, reservas orgânicas, taxa fotossintética e ambiente luminoso (Harris, 1978).

Hodgson (1990) considera que as variáveis do pasto que apresentam maior consistência sobre a produção de forragem são a altura e o índice de área foliar (IAF), especialmente tratando-se de gramíneas forrageiras prostradas, de porte baixo e com alto potencial de perfilhamento. Num valor de IAF chamado ótimo, a interceptação de aproximadamente toda a luz incidente com um mínimo de autosombreamento proporcionaria o máximo valor de taxa de crescimento da cultura (TCC, peso de matéria seca acumulado por unidade de área por unidade de tempo) (Watson, 1958; Brown \& Blaser, 1968; Rhodes, 1973). A interceptação de luz é, no entanto, regulada por uma série de fatores, tais como composição botânica/morfológica do pasto, hábito de crescimento da planta, estrutura do pasto, ângulo foliar e disposição das folhas no dossel do pasto. Este conjunto de características morfológicas e estruturais do pasto determina o grau de atenuação da luz ao atravessar o dossel, ou seja, definem o coeficiente de atenuação da luz (k). Num estudo com gramíneas de diferentes arquiteturas, Sheehy \& Cooper (1973) observaram que os valores de TCC variaram de acordo com a morfologia da planta e com o coeficiente de extinção luminosa.

Apesar de algumas limitações no uso do conceito de IAF no manejo de pastagens, que surgem principalmente por mudanças nas características fotossintéticas, na arquitetura e composição botânica do pasto, Brown \& Blaser (1968) concluíram que o IAF, relacionado à extinção de luz no dossel, parece uma forma útil para entender a produção de forragem e o desenvolvimento de melhores variedades e práticas de manejo.
Este trabalho teve como objetivo avaliar o efeito de diferentes intensidades de pastejo sobre o índice de área foliar, o coeficiente de extinção luminosa, e acúmulo de forragem em pastagens de Cynodon spp. pastejadas por ovinos em regime de lotação contínua.

\section{Material e Métodos}

O experimento foi conduzido na Unidade Experimental de Plantas Forrageiras (UEPF) em área do Departamento de Produção Animal da ESALQ/USP, Piracicaba, SP. O experimento foi instalado em solo classificado como Terra Roxa Estruturada eutrófica, cuja análise de terra revelou os seguintes resultados: $\mathrm{pH}\left(\mathrm{CaCl}_{2}\right)=5,4 ; \mathrm{MO}$ $\left(\mathrm{g} \mathrm{kg}^{-1}\right)=37,0 ; \mathrm{P}\left(\mathrm{mg} \mathrm{dm}^{-3}\right)=99 ; \mathrm{K}\left(\mathrm{cmol}_{\mathrm{c}}\right)=0,46 ; \mathrm{Ca}$ $\left(\mathrm{cmol}_{\mathrm{c}}\right)=7,50 ; \mathrm{Mg}\left(\mathrm{cmol}_{\mathrm{c}}\right)=2,60 ; \mathrm{H}+\mathrm{Al}\left(\mathrm{cmol}_{\mathrm{c}}\right)=3,00$; SB $\left(\mathrm{cmol}_{\mathrm{c}}\right)=10,60 ; \mathrm{T}\left(\mathrm{cmol}_{\mathrm{c}}\right)=13,60$ e V $(\%)=78,0$. Em face da elevada fertilidade do solo, não foram realizadas práticas de correção da fertilidade no momento da instalação do experimento. Durante o período experimental (3 de julho a 31 de dezembro de 1998), a temperatura média diária máxima foi de $30,0^{\circ} \mathrm{C}$, e a mínima, de $10,8^{\circ} \mathrm{C}$. Já a precipitação pluvial total foi de $626,5 \mathrm{~mm}$, e a umidade relativa do ar variou entre 76 e $83 \%$.

O delineamento experimental foi o de blocos completos casualizados, em parcelas subdivididas, com quatro repetições. Os tratamentos corresponderam a combinações entre três cultivares de Cynodon spp. - Tifton 85, Florakirk e Coastcross -, que foram alocadas nas parcelas, e de quatro condições de pasto, geradas por ovinos sob regime de lotação contínua, alocadas nas subparcelas. Cada condição de pasto correspondeu a uma altura média de 5,10 , 15 e $20 \mathrm{~cm}$, mantida constante por meio da adição ou retirada de animais das unidades experimentais durante $o$ transcorrer do experimento. O ensaio apresentou um total de 48 unidades experimentais (4 blocos x 12 unidades/ bloco) com uma área média de $400 \mathrm{~m}^{2}$ cada, separadas por tela de $1,20 \mathrm{~m}$ de altura para delimitação dos piquetes (unidades experimentais) e contenção dos animais. A área experimental total compreendeu 2,0 ha.

Em 3 de junho de 1998 os animais foram alocados nas unidades experimentais que haviam atingido a altura predeterminada para os tratamentos. À medida que as demais unidades experimentais atingiam a altura desejada, os animais eram adicionados dando início ao processo de pastejo. Durante a condução do experimento, de 3 de julho a 31 de dezembro de 1998, foram realizadas quatro adubações nitrogenadas com sulfato de amônio, num total de $155 \mathrm{~kg} \mathrm{ha}^{-1} \mathrm{de} \mathrm{N}$. 
A altura média dos pastos foi monitorada semanalmente, mediante a tomada de 20 leituras em cada unidade experimental com o prato ascendente (rising plate meter) (Frame, 1981), duas vezes por semana. Uma vez ao mês eram tomadas 18 leituras com uma régua (sward stick), de forma concomitante com as leituras do prato ascendente. A partir dos dados, era calculado um fator de conversão entre as alturas do prato e da régua, gerando-se, dessa forma, o valor de altura usado como referência para o monitoramento dos tratamentos. Os animais foram adicionados ou retirados das unidades experimentais, conforme a necessidade de manutenção das alturas determinadas pelos tratamentos.

$\mathrm{O}$ acúmulo de forragem foi medido dentro de gaiolas de exclusão, em número de três por unidade experimental, compreendendo uma área de, aproximadamente, $0,5 \mathrm{~m}^{2}$ $(70 \mathrm{~cm} \times 70 \mathrm{~cm})$ cada, e observando-se um intervalo de 21 dias entre amostragens sucessivas. Após a amostragem, as gaiolas foram rotacionadas nas unidades experimentais e ancoradas em novos pontos representativos da sua condição (altura média) no momento do rodízio. Os cálculos de acúmulo de forragem foram feitos com base no método agronômico da diferença conforme Davies et al. (1993).

Uma vez ao mês era feita a calibração entre altura e massa de forragem, com a finalidade de relacionar a altura média do pasto com a massa de forragem existente e também para o cálculo de acúmulo de matéria seca, minimizando a necessidade de amostragens destrutivas. Foram escolhidos dois pontos de cada unidade experimental onde foram colocados anéis de $0,25 \mathrm{~m}^{2}$ de área procurando sempre o ponto mais baixo e o ponto mais alto a fim de obter a maior amplitude possível de alturas. Foi medida a altura dentro do anel, com a régua e com o prato ascendente; depois, a forragem foi cortada no nível do solo, e lavada, para eliminação de eventuais resíduos de terra e fezes, secada em estufa a $65^{\circ} \mathrm{C}$ até massa constante, e pesada. Assim, após determinada a massa seca, foram estabelecidas equações mediante análise de regressão linear entre altura e massa de forragem, em cada cultivar. A partir dessas equações, os valores de altura do pasto foram transformados em massa de forragem e calculado o acúmulo de matéria seca.

Para avaliação do índice de área foliar (IAF), foram cortados dois quadrados de $25 \mathrm{~cm}$ de lado por unidade experimental, respeitando-se um intervalo mensal entre amostragens. A forragem foi cortada no nível do solo e levada para laboratório no menor espaço de tempo possível. Ao final da colheita, as amostras eram lavadas, subamostradas, e fracionadas manualmente em material vivo e morto. O material vivo foi, depois, fracionado em "folhas" (lâminas foliares) e "hastes" (haste e pseudo-haste). Após a separação destes componentes, uma subamostra das lâminas verdes tinha sua área estimada através de um integrador de área foliar marca LI-COR modelo LI-3100. Posteriormente, toda a forragem era levada para secagem em estufa a $65^{\circ} \mathrm{C}$ até massa constante, juntamente com os demais componentes separados. Após secagem, as amostras eram pesadas. Por meio das relações obtidas entre peso seco e área de folhas, calculou-se a área total de folhas na área de coleta da amostra $\left(625 \mathrm{~cm}^{2}\right.$ quadrado $\left.^{-1}\right)$ e o IAF correspondente $\left(\mathrm{cm}^{2}\right.$ de folhas $\mathrm{cm}^{-2}$ de solo).

Com os dados de IAF e interceptação de luz, calculouse o coeficiente de extinção luminosa (k), conforme descrito por Sheehy \& Cooper (1973) como $\mathrm{k}=\left[\log _{\mathrm{e}}\left(\mathrm{I} / \mathrm{I}_{0}\right)\right] / \mathrm{IAF}$, onde I e $\mathrm{I}_{0}$ são os valores de irradiância abaixo e acima da folhagem, respectivamente.

As avaliações de irradiância foram feitas com um fotômetro LI-COR line (quantum sensor) modelo LI-250. Foram tomadas seis leituras em pontos representativos de cada unidade experimental $(5,10,15 \mathrm{ou} 20 \mathrm{~cm})$. As leituras foram realizadas ao redor das $12 \mathrm{~h}$, sob céu claro, a cada quatro semanas.

Os resultados foram analisados utilizando-se o procedimento GLM do pacote estatístico SAS. Dentro deste procedimento, a análise foi realizada pelo subprocedimento de medidas repetidas no tempo, uma vez que todas as variáveis em estudo foram coletadas ao longo de vários meses (SAS Institute, 1988). Utilizou-se o "LSMEANS" para a comparação de médias entre tratamentos.

\section{Resultados e Discussão}

Os efeitos de mês $(\mathrm{P}=0,0001)$, cultivar $(\mathrm{P}=0,0001)$, altura $(\mathrm{P}=0,0001)$ e interações mês $\mathrm{X}$ cultivar $(P=0,0001)$, cultivar $x$ altura $(P=0,0357)$ no índice de área foliar podem ser observados na Tabela 1. Os valores de IAF encontrados variaram entre 0,21 e 3,71. Estes são inferiores aos relatados por Brougham $(1956,1957)$ com relação a plantas forrageiras de clima temperado, particularmente azevém perene. Esse autor relatou que $95 \%$ de interceptação de luz foi obtido com um IAF igual a 5,0 e 3,5, respectivamente, com relação a azevém e trevo-branco. No entanto, Humphreys (1966) mostrou que os valores de IAF crítico para pastagens situam-se, normalmente, entre 3 e 5 , e que nesta faixa a interceptação da luz (IL) seria de cerca de $95 \%$ da radiação solar incidente. Ensaio conduzido por Clapp 
Tabela 1. Índice de área foliar $\left(\mathrm{cm}^{2}\right.$ de folhas $\mathrm{m}^{-2}$ de solo) em cultivares de Cynodon spp. mantidos em diferentes alturas de pasto durante o período de julho a dezembro de $1998^{(1)}$.

\begin{tabular}{|c|c|c|c|c|c|}
\hline \multirow[t]{2}{*}{ Altura } & \multicolumn{3}{|c|}{ Cultivar } & \multirow[t]{2}{*}{ Média } & \multirow[t]{2}{*}{$\mathrm{EPM}^{(2)}$} \\
\hline & Tifton 85 & Florakirk & Coastcross & & \\
\hline & \multicolumn{5}{|c|}{ Julho } \\
\hline 5 & $1,21 \mathrm{Ca}$ & $0,78 \mathrm{Ba}$ & $0,86 \mathrm{Aa}$ & $0,95 \mathrm{C}$ & 0,16 \\
\hline 10 & $2,13 \mathrm{Ba}$ & $1,61 \mathrm{Aab}$ & $1,24 \mathrm{Ab}$ & $1,66 \mathrm{~B}$ & 0,16 \\
\hline 15 & $2,74 \mathrm{Ba}$ & $1,71 \mathrm{Ab}$ & $1,40 \mathrm{Ab}$ & $1,95 \mathrm{~B}$ & 0,16 \\
\hline 20 & $3,67 \mathrm{Aa}$ & $2,06 \mathrm{Ab}$ & $1,59 \mathrm{Ab}$ & $2,44 \mathrm{~A}$ & 0,16 \\
\hline Média & $2,44 a$ & $1,54 \mathrm{~b}$ & $1,27 b$ & & \\
\hline \multirow[t]{2}{*}{ EPM } & 0,13 & 0,13 & 0,13 & & \\
\hline & \multicolumn{5}{|c|}{ Agosto } \\
\hline 5 & $0,84 \mathrm{Da}$ & $0,70 \mathrm{Ba}$ & $0,50 \mathrm{Ba}$ & $0,68 \mathrm{C}$ & 0,16 \\
\hline 10 & $1,67 \mathrm{Ca}$ & $1,47 \mathrm{ABa}$ & $1,22 \mathrm{ABa}$ & $1,45 \mathrm{~B}$ & 0,16 \\
\hline 15 & $2,66 \mathrm{Ba}$ & $1,33 \mathrm{ABb}$ & $1,00 \mathrm{ABb}$ & $1,67 \mathrm{~B}$ & 0,16 \\
\hline 20 & 3,71 Aa & $2,04 \mathrm{Ab}$ & $1,37 \mathrm{Ab}$ & $2,37 \mathrm{~A}$ & 0,16 \\
\hline Média & $2,22 \mathrm{a}$ & $1,38 b$ & $1,02 b$ & & \\
\hline \multirow[t]{2}{*}{ EPM } & 0,14 & 0,14 & 0,14 & & \\
\hline & \multicolumn{5}{|c|}{ Setembro } \\
\hline 5 & $0,74 \mathrm{Ca}$ & $0,60 \mathrm{Aa}$ & $0,21 \mathrm{Aa}$ & $0,51 \mathrm{C}$ & 0,13 \\
\hline 10 & $1,02 \mathrm{BCa}$ & $0,75 \mathrm{Aa}$ & $0,53 \mathrm{Aa}$ & $0,77 \mathrm{BC}$ & 0,13 \\
\hline 15 & $1,57 \mathrm{Ba}$ & $0,91 \mathrm{Ab}$ & $0,66 \mathrm{Ab}$ & $1,05 \mathrm{~B}$ & 0,13 \\
\hline 20 & $2,67 \mathrm{Aa}$ & $1,08 \mathrm{Ab}$ & $0,78 \mathrm{Ab}$ & $1,51 \mathrm{~A}$ & 0,13 \\
\hline Média & $1,50 \mathrm{a}$ & $0,83 b$ & $0,55 b$ & & \\
\hline \multirow[t]{2}{*}{ EPM } & 0,11 & 0,11 & 0,11 & & \\
\hline & \multicolumn{5}{|c|}{ Outubro } \\
\hline 5 & $0,97 \mathrm{Ca}$ & $0,96 \mathrm{Ba}$ & $0,70 \mathrm{Ba}$ & $0,88 \mathrm{C}$ & 0,11 \\
\hline 10 & $1,92 \mathrm{Ba}$ & $1,58 \mathrm{Aab}$ & $1,30 \mathrm{Ab}$ & $1,60 \mathrm{~B}$ & 0,11 \\
\hline 15 & $2,04 \mathrm{Ba}$ & $1,81 \mathrm{Aa}$ & $1,53 \mathrm{Aa}$ & $1,79 \mathrm{~B}$ & 0,11 \\
\hline 20 & $3,23 \mathrm{Aa}$ & $1,84 \mathrm{Ab}$ & $1,64 \mathrm{Ab}$ & $2,24 \mathrm{~A}$ & 0,11 \\
\hline Média & $2,04 a$ & $1,55 \mathrm{~b}$ & $1,29 \mathrm{~b}$ & & \\
\hline \multirow[t]{2}{*}{ EPM } & 0,10 & 0,10 & 0,10 & & \\
\hline & \multicolumn{5}{|c|}{ Novembro } \\
\hline 5 & $0,89 \mathrm{Ca}$ & $0,77 \mathrm{Aa}$ & $0,68 \mathrm{Aa}$ & $0,78 \mathrm{C}$ & 0,08 \\
\hline 10 & $1,09 \mathrm{Ca}$ & $0,92 \mathrm{Aa}$ & $1,06 \mathrm{Aa}$ & $1,03 \mathrm{~B}$ & 0,08 \\
\hline 15 & $1,51 \mathrm{Ba}$ & $1,01 \mathrm{Ab}$ & $0,93 \mathrm{Ab}$ & $1,15 \mathrm{AB}$ & 0,08 \\
\hline 20 & $2,04 \mathrm{Aa}$ & $1,03 \mathrm{Ab}$ & $1,05 \mathrm{Ab}$ & $1,37 \mathrm{~A}$ & 0,08 \\
\hline Média & $1,38 \mathrm{a}$ & $0,93 b$ & $0,93 b$ & & \\
\hline \multirow[t]{2}{*}{ EPM } & 0,07 & 0,07 & 0,07 & & \\
\hline & \multicolumn{5}{|c|}{ Dezembro } \\
\hline 5 & $0,59 \mathrm{Ba}$ & $0,49 \mathrm{Ba}$ & $0,66 \mathrm{Aa}$ & $0,58 \mathrm{~B}$ & 0,11 \\
\hline 10 & $1,16 \mathrm{Aa}$ & $0,69 \mathrm{Ba}$ & $0,85 \mathrm{Aa}$ & $0,90 \mathrm{~A}$ & 0,11 \\
\hline 15 & $0,97 \mathrm{ABa}$ & $1,40 \mathrm{Aa}$ & $1,11 \mathrm{Aa}$ & $1,16 \mathrm{~A}$ & 0,11 \\
\hline 20 & $1,48 \mathrm{Aa}$ & $0,74 \mathrm{Bb}$ & $1,02 \mathrm{Aab}$ & $1,08 \mathrm{~A}$ & 0,11 \\
\hline Média & $1,05 \mathrm{a}$ & $0,83 a$ & $0,91 \mathrm{a}$ & & \\
\hline EPM & 0,09 & 0,09 & 0,09 & & \\
\hline
\end{tabular}

(1) Médias seguidas de mesma letra, maiúscula na coluna e minúscula na linha, não diferem entre si ( $\mathrm{P}>0,05)$. (2) Erro-padrão da média. 
Junior et al. (1965) revelou valores de IAF entre 1,4 e 2,7 com relação a Cynodon dactylon cv. Coastal semelhantes aos obtidos neste estudo. Em trabalho mais recente, Gomide (1996) observou valores de IAF variando entre 1,68 e 5,85 para Tifton 85, e 1,19 e 5,89 para Florakirk aos 14 e 42 dias de crescimento, respectivamente.

A cultivar Tifton 85 apresentou IAF mais elevado que o de Florakirk, que não diferiu de Coastcross (Tabela 1). As diferenças varietais refletem a história evolutiva da população, sejam as adquiridas naturalmente, ou através de programas de melhoramento e seleção deliberados (Cooper, 1983; Madakadze et al., 1998). Os maiores valores de IAF da Tifton 85 podem ser associados a seu porte mais alto, com hastes grandes e folhas mais largas e compridas que as dos outros híbridos (Burton et al., 1993). Tifton 85 mostrou-se mais sensível a variações em altura do pasto que Coastcross. Os pastos mantidos mais altos apresentaram os maiores valores de IAF, como seria esperado, o que corrobora os resultados encontrados por Clapp Junior et al. (1965). Os menores valores de IAF encontrados nos meses de novembro e dezembro podem ter sido causados pela ocorrência de um período de estiagem bastante severo, com valores pluviométricos no mês de novembro correspondentes à média mais baixa dos últimos 40 anos (26,8 vs. $150 \mathrm{~mm}$ ) (ESALQ, 2000).

A interação entre cultivar e intensidade de pastejo durante o período experimental pode ter sido conseqüência da mudança no ranqueamento dos valores de IAF entre as cultivares com relação às intensidades de pastejo. Ou seja, até o mês de outubro, com maiores intensidades de pastejo $(5$ e $10 \mathrm{~cm})$, os maiores valores de IAF foram apresentados por Tifton 85, seguidos da Florakirk e da Coastcross. A partir de novembro, os valores de IAF de Florakirk foram semelhantes, e depois, superados pela Coastcross, exceção feita para a intensidade de pastejo de $15 \mathrm{~cm}$ no mês de dezembro (Tabela 1). Tais diferenças devem estar relacionadas ao fato de que durante o período de avaliação, particularmente de agosto a novembro, as cultivares Florakirk e Coastcross apresentaram desenvolvimento reprodutivo (florescimento) bastante intenso e contínuo, principalmente nas maiores alturas de pasto, fato este que deve ter sido a causa das variações na estrutura do dossel (e.g. proporção de material morto e material vivo) relacionadas com mudanças nas proporções de haste e folha do pasto (Fagundes, 1999). Humphreys (1966) mencionou que Panicum maximum var. trichoglume também apresentou um florescimento mais intenso em pastos pastejados lenientemente e quando limitações de umidade estiveram aparentes. De acordo com esse autor, o grau de florescimento foi negativamente relacionado à intensidade de desfolha, e as diferenças nos valores de IAF, induzidas pelas desfolhas, foram afetadas pelo florescimento. Segundo Cooper (1983), as diferenças entre e dentro de espécies forrageiras devem-se a algumas características do pasto, como: tamanho da folha, ângulo de inserção entre a folha e o caule, rigidez das folhas, etc., que podem afetar sua estrutura, e, conseqüentemente, o IAF, a IL, e o acúmulo de matéria seca.

Os resultados referentes ao coeficiente de extinção luminosa (k) são mostrados nas Tabelas 2 e 3 . Houve diferenças referentes a mês do ano $(\mathrm{P}=0,0006)$,

Tabela 2. Coeficiente de extinção luminosa (k) em pastos de Cynodon spp. de julho a dezembro de $1998^{(1)}$.

\begin{tabular}{lcccc}
\hline \multirow{2}{*}{ Mês } & \multicolumn{3}{c}{ Cultivar } & \multirow{2}{*}{ EPM $^{(2)}$} \\
\cline { 2 - 4 } & Tifton 85 & Florakirk & Coastcross \\
\hline Julho & $0,88 \mathrm{a}$ & $1,13 \mathrm{a}$ & $1,00 \mathrm{a}$ & 0,09 \\
Agosto & $0,90 \mathrm{~b}$ & $1,26 \mathrm{a}$ & $1,08 \mathrm{~b}$ & 0,09 \\
Setembro & $1,41 \mathrm{~b}$ & $1,94 \mathrm{a}$ & $1,93 \mathrm{a}$ & 0,16 \\
Outubro & $0,85 \mathrm{a}$ & $1,00 \mathrm{a}$ & $0,88 \mathrm{a}$ & 0,08 \\
Novembro & $1,27 \mathrm{a}$ & $1,58 \mathrm{a}$ & $1,29 \mathrm{a}$ & 0,15 \\
Dezembro & $1,23 \mathrm{a}$ & $1,50 \mathrm{a}$ & $1,03 \mathrm{a}$ & 0,18 \\
\hline
\end{tabular}

(1) Médias seguidas de mesma letra minúscula na linha não diferem entre si $(\mathrm{P}>0,05) .{ }^{(2)}$ Erro-padrão da média.

Tabela 3. Coeficiente de extinção luminosa (k) em pastos de Cynodon spp. mantidos em diferentes alturas de julho a dezembro de $1998^{(1)}$.

\begin{tabular}{lccccc}
\hline \multirow{2}{*}{ Mês } & \multicolumn{4}{c}{ Altura de pasto (cm) } & \multirow{2}{*}{ EPM $^{(2)}$} \\
\cline { 2 - 5 } & 5 & 10 & 15 & 20 & \\
\hline Julho & $0,38 \mathrm{c}$ & $0,94 \mathrm{~b}$ & $1,30 \mathrm{a}$ & $1,40 \mathrm{a}$ & 0,11 \\
Agosto & $0,71 \mathrm{~b}$ & $0,77 \mathrm{~b}$ & $1,47 \mathrm{a}$ & $1,37 \mathrm{a}$ & 0,11 \\
Setembro & $0,98 \mathrm{~b}$ & $1,73 \mathrm{a}$ & $2,25 \mathrm{a}$ & $2,07 \mathrm{a}$ & 0,18 \\
Outubro & $0,35 \mathrm{c}$ & $0,73 \mathrm{~b}$ & $1,19 \mathrm{a}$ & $1,35 \mathrm{a}$ & 0,09 \\
Novembro & $0,45 \mathrm{c}$ & $1,08 \mathrm{~b}$ & $1,75 \mathrm{a}$ & $2,23 \mathrm{a}$ & 0,18 \\
Dezembro & $0,10 \mathrm{~b}$ & $0,55 \mathrm{~b}$ & $1,91 \mathrm{a}$ & $2,44 \mathrm{a}$ & 0,21 \\
\hline
\end{tabular}

(1) Médias seguidas de mesma letra minúscula na linha não diferem entre si $(\mathrm{P}>0,05) .{ }^{(2)}$ Erro-padrão da média. 
cultivar ( $\mathrm{P}=0,0020)$, altura $(\mathrm{P}=0,0001)$ e interação mês do ano $\mathrm{x}$ altura $(\mathrm{P}=0,0040)$. A cultivar Florakirk apresentou $\mathrm{k}$ mais elevado do que Tifton $85 \mathrm{e}$ Coastcross em agosto (Tabela 2), sugerindo, assim, que esta cultivar tem tendência a apresentar uma arquitetura foliar mais horizontal (planófila). Siddique et al. (1989) e Yunusa et al. (1993) relataram que um valor mais elevado de $\mathrm{k}$ pode ser atribuído à distribuição mais uniforme da área foliar e à arquitetura mais plana do dossel. Yunusa et al. (1993) evidenciaram que os valores de $\mathrm{k}$ diferiram entre cultivares dentro da estação de crescimento e diferiram na mesma cultivar durante a mesma estação de crescimento, observações, estas, semelhantes às obtidas no presente estudo (Tabela 2).

Pastos mantidos mais altos apresentaram os maiores valores de $\mathrm{k}$, o que provavelmente está associado a folhas maiores e mais horizontais, diminuindo a quantidade de luz propagada ao longo do perfil do dossel (Tabela 3). Estes valores foram aumentando de forma significativa até a altura de $15 \mathrm{~cm}$, e não foram observadas diferenças entre as alturas de $15 \mathrm{e}$ $20 \mathrm{~cm}$. Os valores de $\mathrm{k}$ aumentaram de julho a setembro, sofreram uma redução em outubro, e voltaram a aumentar em dezembro. Tal padrão seguiu o comportamento observado de florescimento das plantas, particularmente da Florakirk e da Coastcross, de forma que no mês de florescimento mais pronunciado (outubro) houve redução nos valores de k. Contrariamente, quando as plantas apresentavam-se em estádio vegetativo nos demais meses, os valores de $\mathrm{k}$ foram mais elevados. Este fato pode ter sido causado pelas mudanças típicas em estrutura do dossel de pastos reprodutivos, caracterizadas pelo aumento da proporção de hastes em relação a folhas, consequiência do processo de elongação das hastes. As cultivares apresentaram comportamento de florescimento diferenciado durante o experimento (Fagundes, 1999), fato que pode ter sido o responsável pela interação mês do ano $\mathrm{x}$ altura de pasto observada.

Diferenças nos valores de $\mathrm{k}$ em relvados formados por diferentes espécies ou cultivares de plantas, mas que possuem um mesmo valor de IAF, são atribuídas à variação na arquitetura das folhas, sendo estas os principais órgãos responsáveis pela interceptação da luz. Os valores de k encontrados no presente experimento variaram entre 0,10 e 2,44
(Tabela 3). A maioria dos trabalhos relata que os valores de k variam entre 0 e 1 (Sheehy \& Cooper, 1973; Hay \& Walker, 1989; Madakadze et al., 1998). No entanto, valores mais elevados de k foram citados por Yunusa et al. (1993), em que um alto k com base na radiação fotossinteticamente ativa $(\mathrm{PAR})\left(\mathrm{k}_{\mathrm{P}}\right)$, de 2,20, foi encontrado em trigo var. Kulin, no estrato de 60 a $80 \mathrm{~cm}$ de altura. Para a maioria das culturas, o k baseado na radiação fotossinteticamente ativa $\left(k_{\mathrm{P}}\right)$ sempre é maior que o $\mathrm{k}$ determinado com a radiação solar total $\left(\mathrm{k}_{\mathrm{T}}\right)$, sugerindo maior atenuação de PAR dentro do dossel quando comparado à radiação solar total. Yunusa et al. (1993) observaram um $\mathrm{k}_{\mathrm{P}}$ de 1,33 para uma cultivar de trigo muito superior ao seu $\mathrm{k}_{\mathrm{T}}$ correspondente $(0,45)$. As avaliações realizadas no presente trabalho foram feitas com base em radiação fotossinteticamente ativa (PAR).

Os resultados referentes ao acúmulo de forragem são mostrados nas Tabelas 4 e 5 . Houve diferenças apenas com relação a mês do ano $(P=0,0001)$. Uma característica dos resultados é o comportamento relativamente uniforme das taxas de acúmulo de forragem nas cultivares com o decorrer da estação de crescimento. Estas aumentaram de agosto a outubro, caíram em novembro, provavelmente como conseqüência dos baixos índices pluviométricos ocorridos, embora estes tenham sido superiores aos apresentados durante os meses de agosto e setembro e aumentaram novamente em dezembro (Tabela 4).

Carnevalli \& Silva (1999) relataram valores de taxa de acúmulo de forragem na Coastcross que variaram de 15,7 a 96,2 $\mathrm{kg} \mathrm{ha}^{-1} \mathrm{dia}^{-1}$ de MS.

Tabela 4. Taxas de acúmulo de matéria seca $\left(\mathrm{kg} \mathrm{ha}^{-1} \mathrm{dia}^{-1}\right)$ em pastos de Cynodon spp. de agosto a dezembro de $1998^{(1)}$

\begin{tabular}{lcccc}
\hline Mês & \multicolumn{3}{c}{ Cultivar } & \multirow{2}{*}{ EPM $^{(2)}$} \\
\cline { 2 - 4 } & Tifton 85 & Florakirk & Coastcross \\
\hline Agosto & $27,8 \mathrm{~b}$ & $37,5 \mathrm{ab}$ & $40,0 \mathrm{a}$ & 4,20 \\
Setembro & $29,2 \mathrm{ab}$ & $24,5 \mathrm{~b}$ & $35,3 \mathrm{a}$ & 2,30 \\
Outubro & $70,6 \mathrm{a}$ & $52,3 \mathrm{a}$ & $70,1 \mathrm{a}$ & 6,90 \\
Novembro & $57,1 \mathrm{a}$ & $38,2 \mathrm{~b}$ & $50,7 \mathrm{ab}$ & 5,20 \\
Dezembro & $104,3 \mathrm{a}$ & $79,2 \mathrm{~b}$ & $84,4 \mathrm{ab}$ & 7,30 \\
\hline \multicolumn{5}{|c}{ Médias seguidas de mesma letra minúscula na linha não diferem entre si } \\
(P>0,05). ${ }^{(2)}$ Erro-padrão da média.
\end{tabular}


No presente ensaio, os valores médios de taxa de acúmulo variaram entre 24,5 e 104,3 $\mathrm{kg} \mathrm{ha}^{-1} \mathrm{dia}^{-1} \mathrm{de}$ MS (Tabela 4). Sheehy \& Cooper (1973), trabalhando com várias espécies forrageiras em condições nãolimitantes de umidade e nutrientes no solo, demonstraram grandes diferenças varietais em acúmulo de forragem, associando essas diferenças com variações na distribuição de luz dentro do dossel e sugerindo que a arquitetura das folhas seria um fator importante na determinação da taxa de crescimento das culturas. No entanto, Humphreys (1966), trabalhando com Panicum maximum var. trichoglume, observou que o crescimento foi independente das intensidades de desfolha estudadas, resultados, esses, semelhantes aos obtidos no presente experimento (Tabela 5). Apesar de não terem sido observadas diferenças $(\mathrm{P}>0,05)$ entre as alturas de pasto estudadas, possivelmente elas existiram. Provavelmente não foram detectadas em razão do método utilizado para determinação do acúmulo de forragem. Segundo Frame (1981), o método baseado em gaiola de exclusão promove uma superestimativa da quantidade de forragem nos pastos mais baixos e uma subestimativa nos pastos mais altos. Isso acontece porque pastos mantidos sempre baixos possuem uma alta quantidade de perfilhos pequenos e uma baixa interceptação de luz. Quando estes pastos são isolados da ação do animal, crescem em tamanho sem haver uma redução proporcional na densidade de perfilhos durante o período de avaliação, ou seja, há um intervalo de tempo entre o sinal de competição por luz e a morte dos perfilhos

Tabela 5. Taxas de acúmulo de matéria seca $\left(\mathrm{kg} \mathrm{ha}^{-1} \mathrm{dia}^{-1}\right)$ em pastos de Cynodon spp. mantidos em diferentes alturas de agosto a dezembro de $1998^{(1)}$.

\begin{tabular}{|c|c|c|c|c|c|}
\hline \multirow[t]{2}{*}{ Mês } & \multicolumn{4}{|c|}{ Altura de pasto $(\mathrm{cm})$} & \multirow[t]{2}{*}{$\mathrm{EPM}^{(2)}$} \\
\hline & 5 & 10 & 15 & 20 & \\
\hline Agosto & $32,2 \mathrm{a}$ & $36,8 \mathrm{a}$ & $30,8 \mathrm{a}$ & $40,6 \mathrm{a}$ & 4,90 \\
\hline Setembro & $29,7 \mathrm{a}$ & $26,7 a$ & $30,4 a$ & $31,8 \mathrm{a}$ & 2,70 \\
\hline Outubro & $66,2 \mathrm{a}$ & $65,3 \mathrm{a}$ & $61,3 a$ & $64,6 \mathrm{a}$ & 8,10 \\
\hline Novembro & $46,6 \mathrm{ab}$ & $38,1 \mathrm{~b}$ & $46,6 \mathrm{a}$ & $63,4 a$ & 6,10 \\
\hline Dezembro & $89,1 \mathrm{a}$ & $90,2 \mathrm{a}$ & $84,3 \mathrm{a}$ & $93,5 \mathrm{a}$ & 8,60 \\
\hline
\end{tabular}

para um novo equilíbrio. Durante esse período, portanto, estimativas de acúmulo são inflacionadas. Já em pastos mais altos, a interceptação de luz encontra-se mais próxima de valores elevados, diferentemente dos pastos mais baixos. Assim, para o mesmo período de acúmulo (21 dias), chega-se à interceptação de $95 \%$ da luz mais cedo, com redução drástica de acúmulo no período.

Hodgson (1990) caracterizou o processo de acúmulo de forragem como sendo o resultado líquido do balanço entre o crescimento (produção de novos tecidos - folhas e hastes) e senescência/morte. Pastos submetidos a regimes de desfolha intensos apresentam pequeno crescimento mas, em contrapartida, baixa senescência. Por outro lado, pastos submetidos a regimes de desfolha lenientes apresentam crescimento mais acelerado que é, em contrapartida, compensado por taxas maiores de senescência e morte de tecidos (Tainton, 1974). Deve existir, portanto, uma amplitude de combinações e possibilidades de regime de desfolha que geram um mesmo equilíbrio, produção de forragem, a partir de balanços diferentes entre crescimento e senescência (Hodgson, 1990). As intensidades de pastejo empregadas no presente experimento podem fazer parte dessa amplitude de possibilidades, o que também resultaria em produções semelhantes de matéria seca, conforme observado na Tabela 5.

O ambiente luminoso e as características estruturais de pastos de Cynodon spp. estudados sofreram influência de cultivar, época do ano e condição do pasto (altura) e acabaram determinando as respostas das plantas forrageiras aos regimes de desfolha impostos. Contudo, pastos mantidos mais altos apresentaram maior IAF, sem, contudo, produzirem mais forragem que pastos mantidos mais baixos. A variação nos valores de $\mathrm{k}$ sugere alterações em arquitetura das plantas e do dossel relacionadas com época do ano, estádio fisiológico das plantas, e cultivar e regime de desfolha. São necessários mais estudos com a finalidade de propiciar o entendimento dos padrões de resposta e estabelecer relações causa-efeito, de forma que parâmetros-guia de manejo do pastejo possam ser estabelecidos clara e objetivamente, visando assegurar a produtividade e longevidade das pastagens cultivadas. 


\section{Conclusões}

1. As taxas de acúmulo de matéria seca são semelhantes para todas as cultivares, independentemente dos regimes de desfolha.

2. Tifton 85 possui o maior valor de IAF para uma mesma altura de pasto comparativamente a Florakirk e Coastcross.

3. Os maiores valores de IAF em Tifton 85 não estão associados com maiores valores de $\mathrm{k}$, indicando uma variação em arquitetura e arranjo das folhas entre as cultivares estudadas.

4. Os valores mais elevados de coeficiente de extinção de luz (k) são observados nos pastos mantidos mais altos, com a cultivar Florakirk.

\section{Agradecimentos}

Aos estagiários do Grupo de Estudo com Plantas Forrageiras, que ajudaram na instalação, condução e análise do experimento; à CAPES, pela concessão da bolsa de estudos, e à FAPESP, pelo financiamento da pesquisa.

\section{Referências}

BROUGHAM, R. M. Effects of intensity of defoliation on regrowth of pastures. Australian Journal of Agriculture Research, Collingwood, v. 7, n. 5, p. 377387,1956 .

BROUGHAM, R. M. Interception of light by the foliage of pure and mixed stands of pasture plants. Australian Journal of Agriculture Research, Collingwood, v. 9, n. 1, p. 39-52, 1957.

BROWN, R. H.; BLASER, R. E. Leaf area index in pasture growth. Herbage Abstracts, Wallingford, v. 38, n. 1, p. 19, Mar. 1968.

BURTON, G. W.; GATES, R. N.; HILL, G. M. Registration of 'Tifton 85' bermudagrass. Crop Science, Madison, v. 33, n. 2, p. 644-645, 1993.

CARNEVALLI, R. A.; SILVA, S. C. da. Validação de técnicas experimentais para avaliação de características agronômicas e ecológicas de pastagens de Cynodon dactylon cv. Coastcross-1. Scientia Agricola, Piracicaba, v. 56, n. 2, p. 489-499, abr./jun. 1999.
CLAPP JUNIOR, J. G.; CHAMBLEE, B. S.; GROSS, H. D. Interrelationships between defoliation systems, morphological characteristics and growth of 'Coastal bermudagrass'. Crop Science, Madison, v. 5, n. 5, p. 468471,1965

COOPER, J. P. Physiological and morphological advances for forage improvement. In: INTERNATIONAL GRASSLAND CONGRESS, 14., 1981, Lexington. Proceedings... Boulder : Westview, 1983. p. 69-76.

DAVIES, D. A.; FURTHERGILL, M.; MORGAN, C. T. Assessment of contrasting perennial ryegrasses and white clover under continuous stocking in the uplands. 5. Herbage production, quality and intake in years 4-6. Grass and Forage Science, Oxford, v. 48, n. 3, p. 213222, 1993.

ESALQ. Departamento de Ciências Exatas. Dados referentes a médias mensais e totais de chuva: Piracicaba, período 1997 a 2000. Disponível: site Campus Luiz de Queiroz. URL: http://www. ciagri. usp. br/ emdabreu/ MEDIAS. txt. Consultado em 04 ago. 2000.

FAGUNDES, J. L. Efeito de intensidades de pastejo sobre o índice de área foliar, interceptação luminosa e acúmulo de forragem em pastagens de Cynodon spp. Piracicaba : ESALQ, 1999. 69 p. Dissertação de Mestrado.

FRAME, J. Herbage mass. In: HODGSON, J.; BAKER, R. D.; DAVIES, A.; LAIDLAW, A. S.; LEAVER, J. D. (Ed.). Sward measurement handbook. Berkshire : British Grassland Society/Grassland Research Institute, 1981. p. $39-69$.

GOMIDE, C. C. C. Algumas características fisiológicas e químicas de cinco cultivares de Cynodon. Jaboticabal : USP, 1996. 100 p. Dissertação de Mestrado.

HARRIS, W. Defoliation as a determinant of the growth, persistence and composition of pasture. In: WILSON, J. R. (Ed.). Plant relations in pastures. East Melbourne : CSIRO, 1978. p. 67-85.

HAY, R. K. M.; WALKER, A. J. An introduction to the physiology of crop yield. Essex : Longman Scientific and Technical, 1989. 292 p.

HODGSON, J. Grazing management: science into practice. Essex : Longman Scientific and Technical, 1990. 203 p.

HUMPHREYS, L. R. Subtropical grass growth. II. Effects of variation in leaf area index in the field. Queensland Journal of Agricultural and Animal Science, Brisbane, v. 23, p. 337-358, 1966. 
JACQUES, A. V. A. Fisiologia do crescimento de plantas forrageiras: área foliar e reservas orgânicas. In: SIMPÓSIO SOBRE MANEJO DE PASTAGENS, 1., 1973, Piracicaba. Anais... Piracicaba : FEALQ, 1973. p. 95-101.

MADAKADZE, I. C.; COULMAN, B. E.; PETERSON, P.; STEWART, K. A.; SAMSON, R.; SMITH, D. L. Leaf area development, light interception, and yield among switchgrass populations in a short-season area. Crop Science, Madison, v. 38, n. 3, p. 827-834, 1998.

PARSONS, A. J. The effects of season and management on the growth of grass swards. In: JONES, M. B.; LAZENBY, A. (Ed.). The grass crop: the physiological basis of production. London : Chapman and Hall, 1988. p. 129-177.

RHODES, I. Relationship between canopy structure and productivity in herbage grasses and its implication for plant breeding. Herbage Abstracts, Wallingford, v. 43, p. 129 $133,1973$.

SAS INSTITUTE (Cary, Estados Unidos). SAS user's guide: release 6. 03. Cary, 1988. 1028 p.

SHEEHY, J. E.; COOPER, J. P. Light interception, photosynthetic activity, and crop growth rate in canopies of six temperate forage grasses. Journal of Applied Ecology, Oxford, v. 10, n. 1, p. 239-250, 1973.
SIDDIQUE, K. H. M.; BELFORD, R. K.; PERRY, M. W.; TENNANT, D. Growth, development and light interception of old and modern wheat cultivars in a Mediterranean-type environment. Australian Journal of Agricultural Research, Collingwood, v. 40, n. 1, p. 473487, 1989.

SILVA, S. C. da; PEDREIRA, C. G. S. Princípios de ecologia aplicados ao manejo de pastagem. In: SIMPÓSIO SOBRE ECOSSISTEMA DE PASTAGENS, 3., Jaboticabal, 1997. Anais... Jaboticabal : FCAV/FUNEP, 1997. p. 1-62.

TAINTON, N. M. A comparison of different pasture rotations. New Zealand Grassland Association Proceedings, Wellington v. 35, p. 204-210, 1974.

WATSON, D. J. The dependence of net assimilation rate on leaf area index. Annals of Botany, London, v. 22, n. 85, p. 37-54, 1958.

YUNUSA, I. A. M.; SIDDIQUE, K. H. M.; BELFORD, R. K.; KARIMI, M. M. Effect of canopy structure on efficiency of radiation interception and use in spring wheat cultivars during pre-anthesis period in a Mediterraneantype environment. Field Crops Research, Wallingford, v. 35 , n. 2, p. 113-122, 1993. 